\title{
High frequency non-invasive stimulation over the spine: Effects on mood and mechanical pain tolerance in normal subjects
}

\author{
A.D. Towell ${ }^{1,2}$, D. Williams ${ }^{2}$ and S.G. Boyd ${ }^{1}$ \\ 'Department of Clinical Neurophysiology, Great Ormond Street Hospital For \\ Children NHS Trust, Great Ormond Street, London and ${ }^{2}$ Department of \\ Psychology, University of Westminster, London, UK \\ Correspondence to: A.D. Towell, Department of Clinical Neurophysiology, \\ Great Ormond Street Hospital For Children NHS Trust, Great Ormond Street, \\ London, WC1N 3JH, UK
}

\begin{abstract}
We investigated the effects of non-invasive high frequency $(625 \mathrm{~Hz})$ stimulation over the spine on mechanical pain tolerance and subjective mood. Sixty healthy subjects were divided equally into three groups receiving either high intensity (250 V), low intensity (3-4 V) or sham electrical stimulation directly over the spinal cord for 30 minutes. Following high intensity stimulation, subjects felt significantly more elated, leisurely and less tense and, contrary to reports on patients with clinical pain, had lower mechanical pain tolerances. There were no correlations between changes in mood and changes in mechanical pain tolerances. These findings contrasted with the lack of any significant differences in mood or mechanical pain tolerances in a second study where 20 subjects received either high intensity or sham stimulation across the left shoulder joint. The results indicate that decreases in mechanical pain tolerance are independent of changes in mood following non-invasive high frequency, high intensity cutaneous stimulation but that both effects are dependent on that stimulation being applied over the spine.
\end{abstract}

Keywords: Mechanical pain tolerance - Mood - Spinal stimulation

\section{INTRODUCTION}

Although invasive spinal cord stimulation is an effective analgesic treatment, it remains an option of last resort in most instances because of the requirement to implant electrodes into the spinal canal, with its associated risk of infection and the possible need for further surgery due to malfunction of the stimulator (Shealy, 1971; Stanton-Hicks and Salamon, 1997). Early attempts at non-invasive electrical stimulation of the CNS in awake humans were unsuccessful because of the unpleasant sensations associated with the stimulus (Frost et al., 1944), but transcutaneous electrical nerve stimulation (TENS) of the peripheral nervous system has proved practicable and has been widely used (Melzack, 1975; Guieu et al., 1991; Walsh et al., 1995).

Recently, a portable peripheral device has been developed in an attempt to stimulate the spinal cord noninvasively in a manner analogous to TENS. Working on the principle that the minimum amplitude of an electrical impulse required to cause an action potential in any class of nerve fibre has to be increased as the pulse width is reduced (Brinley 1974; $\mathrm{Li}$ and Bak, 1976), high voltage (250 volts maximum) transcutaneous stimulation over the cord and surrounding tissue was found to be tolerable when using a short pulse duration $(4 \mu \mathrm{s})$ and high frequency $(625 \mathrm{~Hz})$.

The technique associated with the use of this stimulator has been called transcutaneous spinal electroanalgesia (TSE) because optimal analgesia is achieved only when electrodes are placed on the skin overlying the cord (MacDonald and Coates, 1995). The efficacy of TSE in chronic pain has been demonstrated using a randomised double-blind crossover trial and it was superior to that of TENS (MacDonald and Coates, 1995).

However, when testing normal subjects with TSE for 20 minutes or so in uncontrolled trials, we noticed a pronounced elevation in mood manifested by subjective reports of well being and elation. We hypothesised that changes in mood might be related to the analgesic 
properties of TSE. In a controlled single-blind study, we tested subjective mood and mechanical pain tolerance in normal subjects before and after a 30 minute treatment session with TSE administered over the spine. In a second study, we tested for the effects of TSE administered across the left shoulder joint.

\section{METHOD}

\section{Subjects}

Following ethical committee approval, 60 subjects ( 26 male; age range 18 to 46 years) were randomly assigned to one of three groups and tested with either high intensity TSE, low intensity TSE or placebo (see below). Exclusion criteria were current use of analgesic medication, poor general health (physical or mental) and major physical injury incurred within the preceding year.

\section{Procedure}

All testing was carried out in a sound attenuated cubicle and the Nowlis Mood Adjective Checklist (Nowlis, 1965) was self-administered by the subjects immediately before TSE stimulation (see below). Subjects were required to respond to each of 12 adjectives as either not felt, slightly felt or definitely felt (scored as 1,2 and 3 respectively). The adjectives were 'angry, tensed up, playful, elated, concentrating, tired, energetic, kindly, sad, sceptical, self-centred and leisurely'.

\section{Pain stimulus}

A pressure tolerance meter was used to measure mechanical pain tolerance applied to the nailbed of the middle finger of the right hand. The meter used a $5 \mathrm{~kg}$ gauge calibrated in $1 \mathrm{~g}$ divisions and the method was similar to methods previously described (Fischer, 1988; Ohrbach and Gale, 1989). The pressure was applied through a $1 \mathrm{~mm}$ wide straight edge, positioned such that the edge rested across the lunula of the nail, whilst ensuring that the blade was clear of the eponychium to avoid any possibility of tissue damage. Mechanical pressure was increased at a constant rate by the experimenter, corresponding to approximately $200 \mathrm{~g} . \mathrm{s}^{-1}$, until the subject indicated verbally that the pain had become too much to bear.

\section{TSE stimulus}

A TSE device (APM Ltd) was used to stimulate over the spinal cord via two $4 \times 4 \mathrm{~cm}$ carbon rubber electrodes placed at Cv7 (anode) and T12 (cathode). The TSE output was a rectangular waveform (pulse with $4 \mu \mathrm{s}$ at $625 \mathrm{~Hz}$ ) which was calibrated on a five point scale with a maximum output voltage of $250 \mathrm{~V}$. Subjects were instructed that, during treatment, they might experience some tingling and pulling sensations in the vicinity of their back but that this would not harm them. The controls of the TSE unit were hidden from the subject. In all groups, the intensity of stimulation was gradually increased over a couple of minutes to reach either intensity $5(250 \mathrm{~V})$, intensity $2(3-4 \mathrm{~V})$ or sham (no battery). Subjects were treated for $30 \mathrm{~min}-$ utes once the desired level of stimulation had been reached and were shown a video in an attempt to control their attention in a standardised way. The Nowlis Mood Adjective Checklist and mechanical pain tolerance were recorded in an identical manner to that previously described.

In the second experiment, twenty different subjects (12 male; age range 19 to 37 years) were tested and randomly assigned to one of two groups; ten were given high intensity TSE across the left shoulder joint and were compared with ten subjects receiving sham stimulation in the same region. Mechanical pain tolerance and mood were tested before and after TSE treatment as described previously.

\section{Data processing}

In Experiment 1, to test for between group differences in baseline mood scores, a Kruskal-Wallis 1-Way ANOVA was used. However, to test for the effects of TSE on mood, each subject was used as their own control and data were transformed into change scores (score after-score before) indicating whether an emotion was being felt more strongly, a positive score, or whether an emotion was being felt less strongly, a negative score. Mood change data were then subjected to Kruskal-Wallis ANOVA. To test for between-group differences in mechanical pain tolerance, data were subjected to 1-Way ANOVA and then processed in the same way as the mood scores (score after - score before) but expressed as a percentage change with positive scores reflecting an increase in mechanical pain tolerance following TSE treatment. One-way ANOVA was used to test for differences in percentage change values. In Experiment 2, mood and pain tolerance data were handled in an identical way, except that Mann-Whitney and t-tests were used in place of Kruskal-Wallis and ANOVA, respectively.

\section{RESULTS}

There was no significant differences in baseline mood scores across the three treatment groups (Maximum Chi-Square $=2.62 ; p>0.05)$. The effect of a 30 minute treatment with TSE was for subjects to report 
TABLE I. Mean change values of emotions following TSE. Positive values represent an emotion being felt more strongly. Following high intensity TSE, subjects felt significantly more elated $(* \star \star=p<0.001)$, leisurely $\left({ }^{*}=p<0.05\right)$ and less tensed up $\left({ }^{*}=p<0.01\right)$

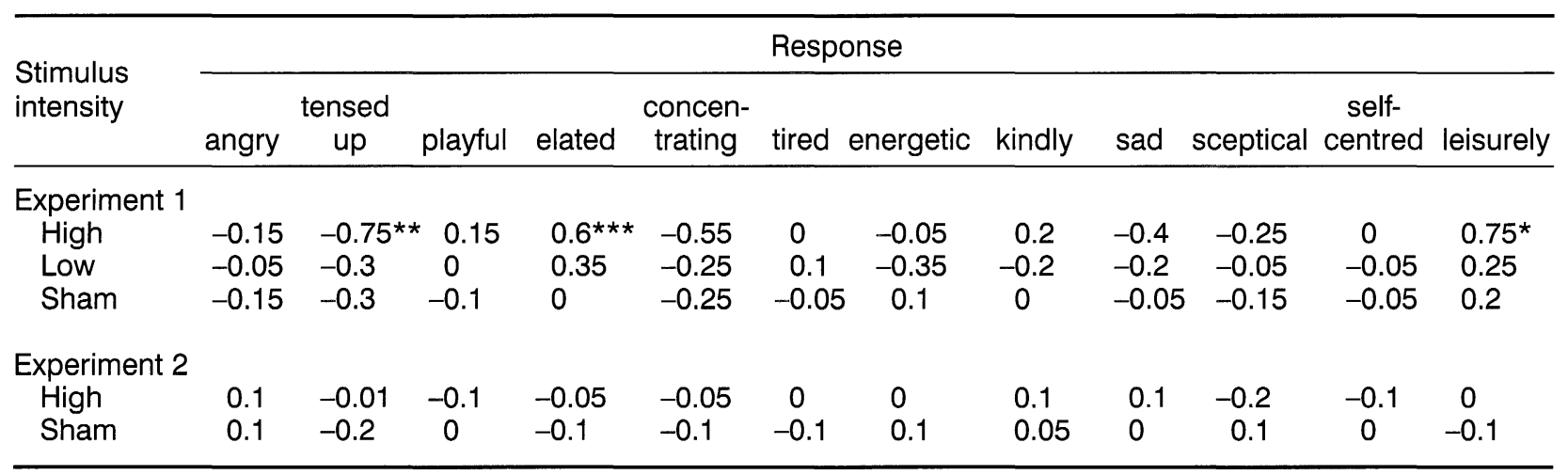

being less 'tensed up' (Chi-Square $=7.7 ; p=0.021$ ), more 'elated' (Chi-Square $=10.9 ; p=0.004$ ) and more 'leisurely' (Chi-Square $=10.0 ; p=0.007$; Table I). These significant changes in mood ('tensed-up', 'elated' and 'leisurely') were seen following high intensity TSE treatment only (Mann Whitney $\mathrm{U}=114.5,95.0$ and 122.5; $p=0.0088,0.0009$ and 0.0167 , respectively).

There were no significant differences in baseline pain tolerance scores across the treatment groups $(\mathrm{F}(2,59)=0.47 ; p=0.627)$. However, there were significant intensity dependent reductions in mechanical pain tolerance following TSE treatment $(\mathrm{F}(2,29)=$ 7.1; $p=0.002$; Table II). Scheffe contrasts revealed a significant difference between high intensity TSE with comparison to sham treatment $(p<0.05)$.

To test for associations between the changes in mood and changes in mechanical pain tolerance, we performed Spearman correlations between these measures in the high intensity TSE treatment group. There were no significant correlations, suggesting separate mechanisms for changes in mood and reductions in mechanical pain tolerance.

In Experiment 2, the baseline measures of mood and mechanical pain tolerance were similar to those recorded in experiment 1 (Table II). However, there were no significant changes in mood (Table I) or in mechanical pain tolerance (Table II) following TSE applied across the left shoulder joint for 30 minutes.

\section{DISCUSSION}

Our results show that high intensity TSE treatment administered over the spinal cord has significant effects on mood consistent with the subjects being more 'elated', 'leisurely' and less 'tensed up'. These results confirm our personal experience with TSE in normal volunteers. However, the prevailing circumstances in acute and chronic clinical pain syndromes are clearly very different, both from each other and from normal controls. It remains to be seen whether mood changes are a significant factor in the claimed analgesic effects of TSE.

Changes in affective behaviour and improvements in memory have been reported in patients with Alzheimer's disease following 30 minute daily treatment sessions with BURST-TENS administered between $\mathrm{T} 1$ and $\mathrm{T} 5$ across the spinal column; patients

TABLE II. Mean (SD) mechanical tolerance values (gms) before and after TSE and mean (SE) percentage change. There was a significant reduction in pain tolerance following high intensity TSE in comparison to sham. $\left({ }^{*}=p<0.05\right)$.

\begin{tabular}{lccc}
\hline Stimulus intensity & \multicolumn{3}{c}{ Mechanical tolerance values } \\
\cline { 2 - 4 } & Before & After & \% change \\
\hline Experiment 1 & & & $-10.94(2.0)^{*}$ \\
High & $1767.3(663.7)$ & $1560.8(578.4)$ & $-6.07(1.0)$ \\
Low & $1602.7(498.1)$ & $1505.7(482.3)$ & $0.01(2.7)$ \\
Sham & $1753.5(611.3)$ & $1726.3(538.7)$ & $0.78(3.7)$ \\
Experiment 2 & & & $-2.09(3.1)$ \\
$\quad$ High & $1792.5(710.1)$ & $1754.5(583.2)$ & $1784.7(562.7)$ \\
Sham & $1839.6(621.4)$ & & \\
\hline
\end{tabular}


felt less dejected and gloomy and more active and alert (Scherder et al., 1992; Scherder et al., 1995). No data on normal controls were reported but it has been argued that the changes in memory and affect are mediated by the hippocampus and hypothalamus following activation of brainstem structures, such as the locus coeruleus and dorsal raphe nucleus (Scherder $e t$ al., 1995).

The mechanism of action of TENS involves peripheral activation of A beta fibres (Howson, 1978) and, possibly, a more central activation of CNS structures (Wolf, 1978; Golding et al., 1986). MacDonald and Coates (1995) suggested that the absence of paraesthesiae implied that A beta fibres are not activated using TSE. However, they did report a 'spinal cord sensation' during TSE treatment $(4 \mu \mathrm{s} ; 100 \mathrm{~Hz})$; they describe this as a "painless feeling of continuous light pressure ... that could be felt deeply in the spinal region". In the present study, because of the considerable subject demands associated with completion of the Nowlis Mood Adjective List, no formal assessment was made of unsolicited comments made by subjects during debriefing. However, some subjects in both treatment groups in the first experiment did report sensations in the vicinity of the cord which described as 'pulling' or 'touching' and this highlights the need for future studies to use double-blinded designs. On the basis of subjective reports it is, therefore, plausible that large fibre pathways are activated in the vicinity of the cord during high intensity TSE. This hypothesis is consistent with animal studies of invasive spinal cord stimulation demonstrating sole recruitment of lowthreshold, large myelinated fibres (Linderoth et al., 1991).

The fact that TSE applied across the left shoulder joint did not produce any changes in mood and mechanical pain tolerance and that TSE over the spinal cord did, suggests that one mechanism of action of TSE involves activation of superficial structures in the vicinity of the cord. Although the pain relieving effects of epidural spinal cord stimulation are thought to be related to stimulation of the dorsal column (Tomita et al., 1996), it remains to be seen whether TSE has any effects on deeper structures within the cord.

The reduction in mechanical pain tolerance following high intensity TSE was unexpected. When used to treat clinical pain, TSE tends to increase mechanical pain thresholds at the sites found to be abnormally tender to palpation. However, in a group of 16 patients with unilateral pain, treatment with TSE increased thresholds at painful sites but reduced them at contralateral non-painful sites (MacDonald and
Coates, 1995). Our results, taken together with MacDonald and Coates (1995), suggest that an increase in pain threshold and reduction in pain tolerance may be a possible mechanism of TSE.

We hypothesised that changes in mechanical pain tolerance would be associated with changes in affective mood but found no correlation between them. On this basis, we must assume that the mood changes and pain tolerance changes are mediated via different mechanisms, though the physiological basis of either effect remains unclear. However, the presence of two different mechanisms seems to exclude some possibilities based on a single system, such as the release of opioids, since changes in analgesia would be anticipated in association with changes in mood (Yaster and Maxwell, 1993).

Several models of spinal cord stimulation suggest the analgesic effects are mediated by a number of neurotransmitter systems, including opiates, serotonin, noradrenaline, substance $\mathrm{P}$ and GABA (Linderoth $e t$ al., 1991). However, in animal models of electroacupuncture, the antinociceptive effect is thought to be mediated by opiate mechanisms at low frequencies and by non-opioid neurotransmitters at high frequencies (Lee and Beitz, 1992). There is also evidence of widespread brainstem activation following both high and low frequency electroacupuncture (Lee and Beitz, 1993). These data suggest a possible model for the mechanism of action of TSE and further studies are required to examine the time course of both its mood enhancing and analgesic properties.

However, the present findings indicate that decreases in mechanical pain tolerance are independent of changes in mood following noninvasive high frequency, high intensity cutaneous stimulation but that both effects are dependent on that stimulation being applied over the spine. The results of the present single-blinded study, taken together with the doubleblinded study of MacDonald and Coates (1995), argue against the effects of TSE being purely placebo and warrant further investigations to elucidate its mechanism of action.

\section{Acknowledgements}

We would like to thank Dr Alex MacDonald for the loan of the TSE stimulator and for his enthusiastic encouragement. Mr Jon Graves is also acknowledged for his assistance in running Experiment 1.

\section{REFERENCES}

Brinley FJ (1974) Excitation and conduction in nerve fibres. In: Medical Physiology (Ed VB Mountcastle) Volume 1, pp. 34-76, CV Mosby Co, St Louis. 
Fischer AA (1988) Documentation of myofascial trigger points. Archives of Physical Medicine and Rehabilitation, 69, 286-291.

Frostig JP, van Harreveld A, Reznick S, Tyler DB and Wiersma CAG (1944) Electronarcosis in animals and man. Archives of Neurology and Psychiatry, 51, 232-242.

Golding JF, Ashton H, Marsh R and Thompson JW (1986) Transcutaneous electrical nerve stimulation produces variable changes in somatosensory evoked potentials, sensory perception and pain threshold: Clinical implications for pain relief. Journal of Neurology, Neurosurgery and Psychiatry, 49, 1397-1406

Guieu R, Tardt-Gervet M and Roll J (1991) Analgesic effects of vibration and transcutaneous electrical nerve stimulation applied separately and simultaneously to patients with chronic pain. Canadian Journal of the Neurologic Sciences, 18, 113-119.

Howson DC (1978) Peripheral neural excitability. Implications for transcutaneous electrical nerve stimulation. Physical Therapy, 58, 1467-1473.

I.ee JH and Beitz AJ (1992) Electroacupuncture modifies the expression of c-fos in the spinal cord induced by noxious stimulation. Brain Research, 577, 80-91.

Lee JH and Beitz AJ (1993) The distribution of brain-stem and spinal cord nuclei associated with different frequencies of electroacupuncture analgesia. Pain, 52, 11-28.

Li CL and Bak A (1976) Excitability characteristics of the A- and C- fibres in a peripheral nerve. Experimental Neurology, 50, 67-69.

Linderoth B, Fedorcsak I and Meyerson BA (1991) Peripheral vasodilatation after spinal cord stimulation: animal studies of putative effector mechanisms. Neurosurgery, 28, 187-195.

MacDonald AJR and Coates TW (1995) The discovery of transcutaneous spinal electroanalgesia and its relief of chronic pain. Physiotherapy, 81, 653-661.

Melzack R (1975) Prolonged relief of pain by brief intense transcutaneous somatic stimulation. Pain, 1, 357-373.

Nowlis V (1965) Research with mood adjective checklist. In: Affect, Cognition and Personality (Eds SS Tomkins and
C Izard), pp. 352-389. Springer, New York.

Ohrbach R and Gale EN (1989) Pressure pain thresholds, clinical assessment, and differential diagnosis: Reliability and validity in patients with myogenic pain. Pain, 39, 157-169.

Scherder EJA, Bouma A and Steen AM (1992) Influence of transcutaneous electrical nerve stimulation on memory in patients with dementia of the Alzheimer type. Journal of Clinical and Experimental Neuropsychology, 14, 951-960.

Scherder EJA, Bouma A and Steen AM (1995) Effects of simultaneously applied short-term transcutaneous electrical nerve stimulation and tactile stimulation on memory and affective behaviour of patients with probable Alzheimers disease. Behavioural Neurology, 8, 3-13.

Shealy CN (1971) Dorsal column electroanalgesia. In : The Textbook of Pain (Eds PD Wall and R Melzack), pp. 906-919. Churchill Livingstone, Edinburgh.

Stanton-Hicks M and Salamon J (1997) Stimulation of the central and peripheral nervous system for the control of pain. Journal of Clinical Neurophysiology, 14, 46-62.

Tomita M, Shimoji K, Denda S, Tobita T, Uchiyama S and Baba H (1996) Spinal tracts producing slow components of spinal cord potentials evoked by descending volleys in man. Electroencephalography and Clinical Neurophysiology, 100, 68-73.

Walsh DM, Liggett C, Baxter D and Allen JM (1995) A double-blind investigation of the hypoalgesic effects of transcutaneous electrical nerve stimulation upon experimentally induced ischemic pain. Pain, 61, 39-45.

Wolf SL (1978) Perspectives on central nervous system responsiveness to transcutaneous electrical nerve stimulation (TENS). Pain, 11, 37-47.

Yaster M and Maxwell L (1993) Opioid agonists and antagonists. In: Pain in Infants, Children and Adolescents (Eds N. Schechter, C Berde and M Yaster), pp. 145-171, Williams and Wilkins.

(Received 6 December 1996; accepted as revised 27 May 1997) 


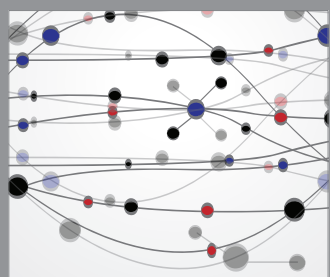

The Scientific World Journal
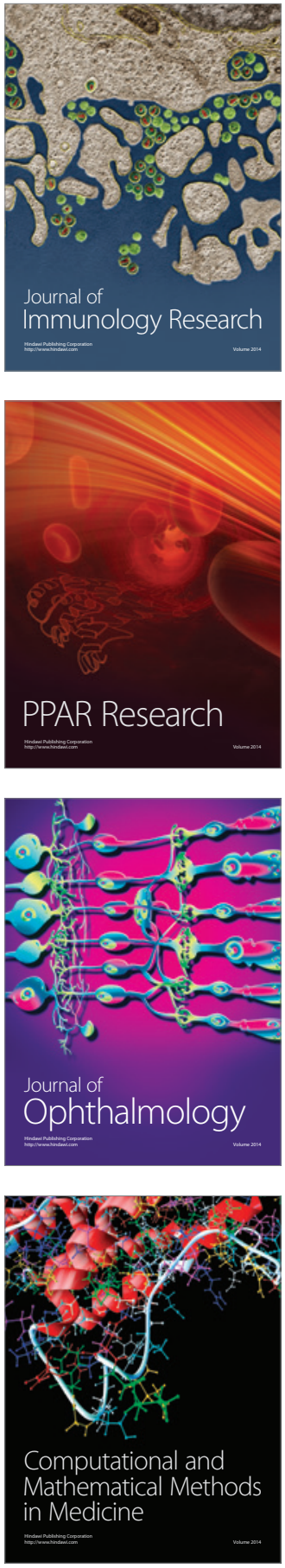

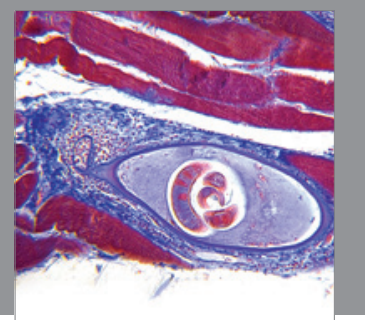

Gastroenterology

Research and Practice
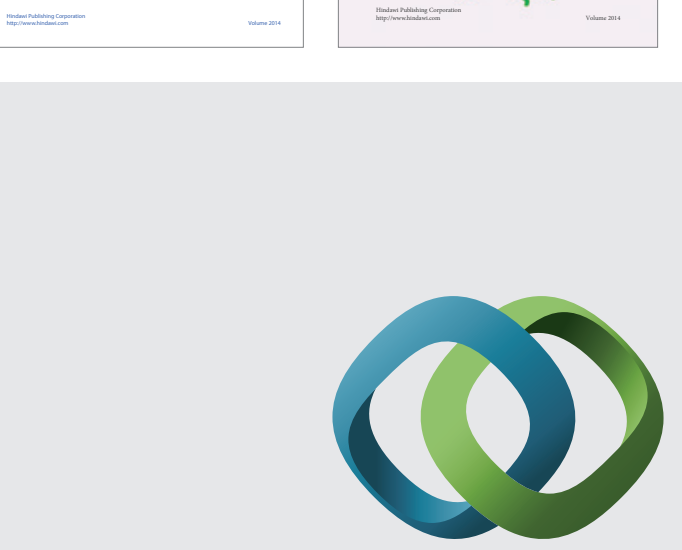

\section{Hindawi}

Submit your manuscripts at

http://www.hindawi.com
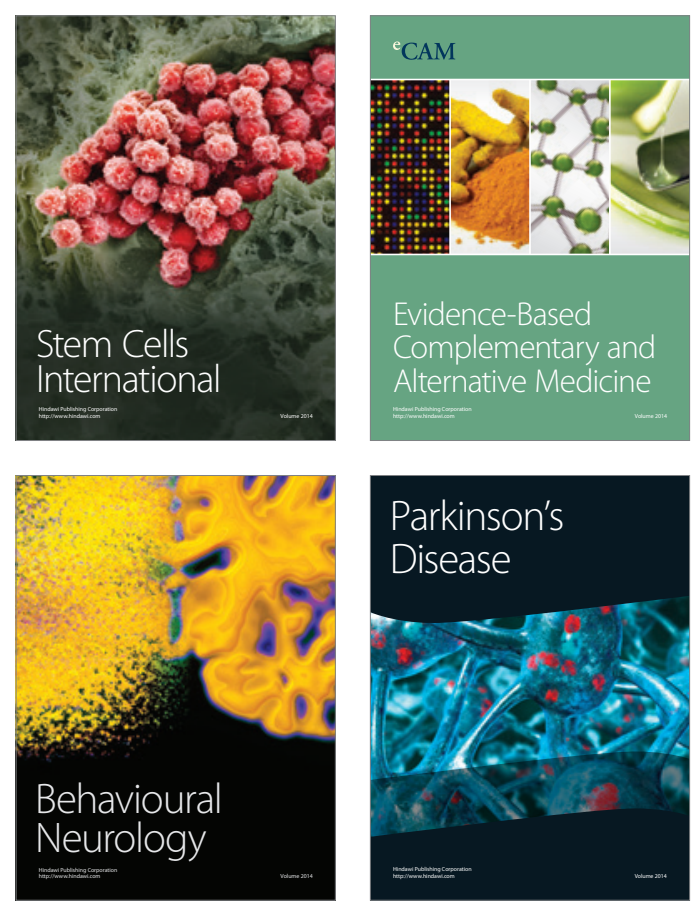

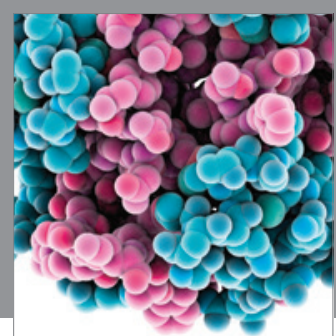

Journal of
Diabetes Research

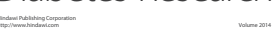

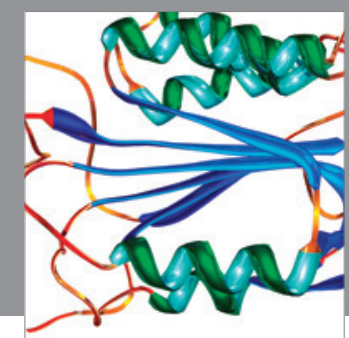

Disease Markers
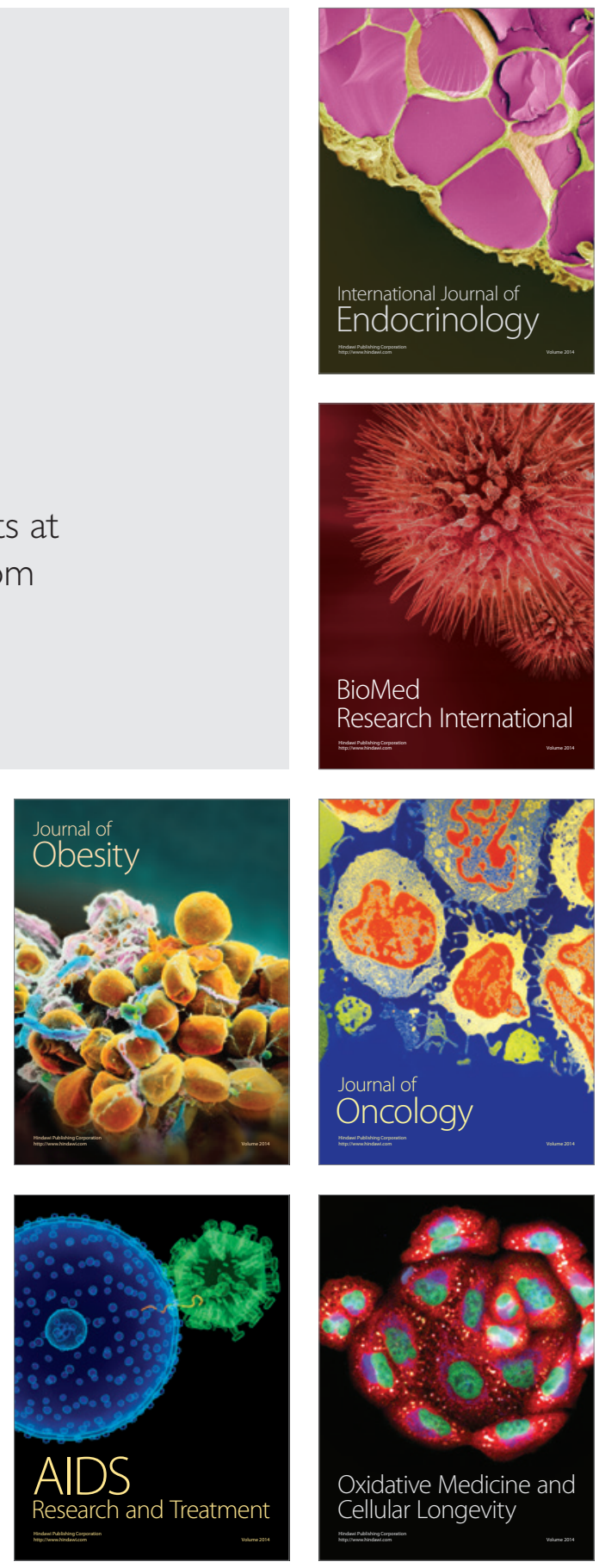